University of Wollongong

Research Online

Faculty of Arts, Social Sciences and Humanities

- Papers

Faculty of Arts, Social Sciences \& Humanities

January 2020

\title{
An Intervention for Mental Health Literacy and Resilience in Organized Sports
}

Stewart A. Vella

University of Wollongong, stvella@uow.edu.au

Christian Swann

Marijka Batterham

University of Wollongong, marijka@uow.edu.au

Katherine Boydell

Simon Eckermann

University of Wollongong, seckerma@uow.edu.au

See next page for additional authors

Follow this and additional works at: https://ro.uow.edu.au/asshpapers

Research Online is the open access institutional repository for the University of Wollongong. For further information contact the UOW Library: research-pubs@uow.edu.au 


\title{
An Intervention for Mental Health Literacy and Resilience in Organized Sports
}

\begin{abstract}
PURPOSE: In this study we tested the effectiveness of a multi-component sports-based program aimed at promoting early intervention, help-seeking and resilience among a sample of adolescent male sport participants. METHODS: The Ahead of the Game program comprised four intervention components and a messaging campaign. Two components targeted mental health literacy, intentions to seek and provide help, and resilience among adolescent males. A mental health literacy program for parents, and a coach education program to assist in the support of athletes' psychological needs were also included. We evaluated the program using a non-randomised controlled trial matching two regional communities. In total, 350 sport participants ( $M=14.53$ years) were included in an intervention group, while $466(\mathrm{M}=$ 14.66 years) received usual practice in a matched control community. One hundred and eighty parents or caregivers and eight coaches also participated in the intervention components. Between-group mean differences on the primary and secondary outcomes were analysed using linear mixed models, adjusted for clustering at club level, participant age, and socioeconomic status. RESULTS: Significant positive group by time interactions were found for the primary outcomes of depression and anxiety literacy, intentions to seek help from formal sources, confidence to seek mental health information, and resilience. We also found a significant positive group by time interaction for the secondary outcome of wellbeing. There were no group by time interactions for social distance (stigma), intentions to seek help from informal sources, implicit beliefs about adversity, perceived familial support, or psychological distress. CONCLUSION: Given the high rates of sport participation worldwide and the increasing focus on mental health in this domain, translation and dissemination of the program may be warranted following replication.
\end{abstract}

\section{Publication Details}

Vella, S. A., Swann, C., Batterham, M., Boydell, K. M., Eckermann, S., Ferguson, H., Fogarty, A., Hurley, D., Liddle, S. K., Lonsdale, C., Miller, A., Noetel, M., Okely, A. D., Sanders, T., Schweickle, M. J., Telenta, J. \& Deane, F. P. (2020). An Intervention for Mental Health Literacy and Resilience in Organized Sports. Medicine and science in sports and exercise,

\section{Authors}

Stewart A. Vella, Christian Swann, Marijka Batterham, Katherine Boydell, Simon Eckermann, Helen Ferguson, Andrea Fogarty, Diarmuid Hurley, Sarah Liddle, Chris Lonsdale, Andrew Miller, Michael Noetel, Anthony D. Okely, Taren Sanders, Matthew Schweickle, Joanne Telenta, and Frank P. Deane 


\section{Medicine \& Science \\ IN \\ Sports \& Exercise}

The Otficial Joumal of the American College of Sports Medicine

. . . Published ahead of Print

\section{An Intervention for Mental Health Literacy and Resilience in Organized Sports}

Stewart A. Vella ${ }^{1}$, Christian Swann ${ }^{2,3}$, Marijka Batterham ${ }^{4}$, Katherine M. Boydell ${ }^{5}$, Simon Eckermann ${ }^{6}$, Helen Ferguson ${ }^{7}$, Andrea Fogarty ${ }^{8}$, Diarmuid Hurley ${ }^{9}$, Sarah K. Liddle ${ }^{10}$, Chris Lonsdale ${ }^{11}$, Andrew Miller ${ }^{12}$, Michael Noetel ${ }^{13}$, Anthony D. Okely ${ }^{14}$, Taren Sanders ${ }^{15}$, Matthew J. Schweickle ${ }^{16}$, Joanne Telenta ${ }^{17}$, Frank P. Deane ${ }^{18}$

${ }^{1}$ School of Psychology, Faculty of Social Sciences, University of Wollongong, Northfields Avenue, Wollongong, Australia; ${ }^{2}$ School of Health and Human Sciences, Southern Cross University, Coffs Harbour, Australia; ${ }^{3}$ Centre for Athlete Development, Experience \& Performance, Southern Cross University, Coffs Harbour, Australia; ${ }^{4}$ School of Mathematics and Applied Statistics, Faculty of Engineering and Information Sciences, University of Wollongong, Wollongong, Australia; ${ }^{5}$ Black Dog Institute, University of New South Wales, Randwick, Australia; ${ }^{6}$ Australian Health Services Research Institute, Sydney Business School, Faculty of Business, University of Wollongong, Northfields Avenue,

Wollongong, Australia; ${ }^{7}$ English Institute of Sport, The High Performance Centre, Bisham Abbey National Sports Centre, Bisham, Buckinghamshire, United Kingdom; ${ }^{8}$ Black Dog Institute, University of

New South Wales, Hospital Road, Randwick, Australia; ${ }^{9}$ School of Psychology, Faculty of Social

Sciences, University of Wollongong, Northfields Avenue, Wollongong, Australia; ${ }^{10}$ School of Psychology, Faculty of Social Sciences, University of Wollongong, Wollongong, Australia; ${ }^{11}$ Institute for

Positive Psychology and Education, Faculty of Health Sciences, Australian Catholic University, North

Sydney, Australia; ${ }^{12}$ School of Education, University of Newcastle, Callaghan NSW, Australia; ${ }^{13}$ Institute for Positive Psychology and Education, Faculty of Health Sciences, Australian Catholic University, North

Sydney, Australia; ${ }^{14}$ Early Start, Faculty of Social Sciences, University of Wollongong, Wollongong,

Australia; ${ }^{15}$ Institute for Positive Psychology and Education, Faculty of Health Sciences, Australian

Catholic University, North Sydney, Australia; ${ }^{16}$ School of Psychology, Faculty of Social Sciences, University of Wollongong, Wollongong, Australia; ${ }^{17}$ Centre for Health and Social Research, St. Patrick's Campus, Melbourne, Australia; ${ }^{18}$ Illawarra Institute for Mental Health, School of Psychology, Faculty of Social Sciences, University of Wollongong, Northfields Avenue, Wollongong, Australia

Accepted for Publication: 27 May 2020

Medicine \& Science in Sports \& Exercise ${ }_{\circledast}$ Published ahead of Print contains articles in unedited manuscript form that have been peer reviewed and accepted for publication. This manuscript will undergo copyediting, page composition, and review of the resulting proof before it is published in its final form. Please note that during the production process errors may be discovered that could affect the content. 


\title{
An Intervention for Mental Health Literacy and Resilience in Organized Sports
}

\author{
Stewart A. Vella ${ }^{1}$, Christian Swann ${ }^{2,3}$, Marijka Batterham ${ }^{4}$, Katherine M. Boydell ${ }^{5}$, \\ Simon Eckermann ${ }^{6}$, Helen Ferguson ${ }^{7}$, Andrea Fogarty ${ }^{8}$, Diarmuid Hurley ${ }^{9}$, Sarah K. Liddle ${ }^{10}$, \\ Chris Lonsdale $^{11}$, Andrew Miller ${ }^{12}$, Michael Noetel ${ }^{13}$, Anthony D. Okely ${ }^{14}$, Taren Sanders ${ }^{15}$, \\ Matthew J. Schweickle ${ }^{16}$, Joanne Telenta ${ }^{17}$, Frank P. Deane ${ }^{18}$
}

${ }^{1}$ School of Psychology, Faculty of Social Sciences, University of Wollongong, Northfields Avenue, Wollongong, Australia; ${ }^{2}$ School of Health and Human Sciences, Southern Cross University, Coffs Harbour, Australia; ${ }^{3}$ Centre for Athlete Development, Experience \& Performance, Southern Cross University, Coffs Harbour, Australia; ${ }^{4}$ School of Mathematics and Applied Statistics, Faculty of Engineering and Information Sciences, University of Wollongong, Wollongong, Australia; ${ }^{5}$ Black Dog Institute, University of New South Wales, Randwick, Australia; ${ }^{6}$ Australian Health Services Research Institute, Sydney Business School, Faculty of Business, University of Wollongong, Northfields Avenue, Wollongong, Australia; ${ }^{7}$ English Institute of Sport, The High Performance Centre, Bisham Abbey National Sports Centre, Bisham, Buckinghamshire, United Kingdom; ${ }^{8}$ Black Dog Institute, University of New South Wales, Hospital Road, Randwick, Australia; ${ }^{9}$ School of Psychology, Faculty of Social Sciences, University of Wollongong, Northfields Avenue, Wollongong, Australia; ${ }^{10}$ School of Psychology, Faculty of Social Sciences, University of Wollongong, Wollongong, Australia; ${ }^{11}$ Institute for Positive Psychology and Education, Faculty of Health Sciences, Australian Catholic University, North Sydney, Australia; ${ }^{12}$ School of Education, University of Newcastle, Callaghan NSW, 
Australia; ${ }^{13}$ Institute for Positive Psychology and Education, Faculty of Health Sciences, Australian Catholic University, North Sydney, Australia; ${ }^{14}$ Early Start, Faculty of Social Sciences, University of Wollongong, Wollongong, Australia; ${ }^{15}$ Institute for Positive Psychology and Education, Faculty of Health Sciences, Australian Catholic University, North Sydney, Australia; ${ }^{16}$ School of Psychology, Faculty of Social Sciences, University of Wollongong, Wollongong, Australia; ${ }^{17}$ Centre for Health and Social Research, St. Patrick's Campus, Melbourne, Australia; ${ }^{18}$ Illawarra Institute for Mental Health, School of Psychology, Faculty of Social Sciences, University of Wollongong, Northfields Avenue, Wollongong, Australia

Corresponding Author: Stewart A. Vella; School of Psychology, Faculty of Social Sciences, University of Wollongong, Northfields Avenue, Wollongong, Australia, 2522 (t) +61 2 42215516; (e) stvella@uow.edu.au.

Funding: The Ahead of the Game study was funded by the Movember Foundation through an Australian Mental Health Initiative grant. The project also received funding from the University of Wollongong Global Challenges Program. Conflicts of Interest: Dr Vella and Dr Swann currently consult to the Movember Foundation on related projects. All other authors declare that they have no conflict of interest. 
Copyright (C) 2020 the Author(s). Published by Wolters Kluwer Health on behalf of the American College of Sports Medicine. This is an open-access article distributed under the terms of the Creative Commons Attribution-Non Commercial-No Derivatives License 4.0 (CCBY-NC-ND), where it is permissible to download and share the work provided it is properly cited. The work cannot be changed in any way or used commercially without permission from the journal. 


\begin{abstract}
Purpose: In this study we tested the effectiveness of a multi-component sports-based program aimed at promoting early intervention, help-seeking and resilience among a sample of adolescent male sport participants. Methods: The Ahead of the Game program comprised four intervention components and a messaging campaign. Two components targeted mental health literacy, intentions to seek and provide help, and resilience among adolescent males. A mental health literacy program for parents, and a coach education program to assist in the support of athletes' psychological needs were also included. We evaluated the program using a non-randomised controlled trial matching two regional communities. In total, 350 sport participants $(M=14.53$ years) were included in an intervention group, while $466(M=14.66$ years $)$ received usual practice in a matched control community. One hundred and eighty parents or caregivers and eight coaches also participated in the intervention components. Between-group mean differences on the primary and secondary outcomes were analysed using linear mixed models, adjusted for clustering at club level, participant age, and socioeconomic status. Results: Significant positive group by time interactions were found for the primary outcomes of depression and anxiety literacy, intentions to seek help from formal sources, confidence to seek mental health information, and resilience. We also found a significant positive group by time interaction for the secondary outcome of wellbeing. There were no group by time interactions for social distance (stigma), intentions to seek help from informal sources, implicit beliefs about adversity, perceived familial support, or psychological distress. Conclusion: Given the high rates of sport participation worldwide and the increasing focus on mental health in this domain, translation and dissemination of the program may be warranted following replication. Key Words: helpseeking; mental health literacy; resilience; wellbeing.
\end{abstract}




\section{INTRODUCTION}

Mental health problems are one of the greatest contributors to the global burden of disease among young people [1]. Half of all mental health problems have their onset before the age of 14 years [2], and the personal and social costs of such problems can last decades. The lifetime prevalence of any mental illness is approximately $22 \%$ among US adolescents, with the median age of onset for mood disorders approximately 13 years [3]. Young men may be at particular risk of suffering the personal and social costs of early onset mental health problems. For example, adherence to traditionally masculine norms has a negative impact on help-seeking, contributing to treatment delays [4]. As such, there is a need to support gender-sensitive evidence-based policies and programs aimed at promoting young men's health [5].

Outside of school, the most time-consuming organised activity for young men is organised sports. Participation in organised sports during childhood and adolescence is over $40 \%$ in most countries worldwide, with participation much higher in high-income countries [6]. In Australia, $75 \%$ of all children and adolescents participate in organised sports in any given year [7]. Further, adolescent males spend, on average, more than eight hours per week in organised sports [8]. Recent policy recommendations regarding young men's mental health care has highlighted organised sport as a priority area to engage young men who do not follow traditional help-seeking pathways, which may improve early intervention efforts and prevention [9]. As such, sports settings are seen as the vehicle through which programming can be delivered to facilitate engagement with traditional forms of mental health care. However, a review of applied sports-based mental health programming shows that it is rarely evidence-based and has little data 
to support efficacy [10]. A systematic review of research shows that studies in this area have typically been of low quality [11].

Organised sports, especially among young people, have the potential to be an engaging vehicle for interventions targeting mental health literacy [11] and resilience [12]. Mental health literacy refers to one's knowledge and beliefs about mental health that influence the recognition, management, and prevention of mental health disorders [13]. Mental health literacy includes knowledge of preventative strategies regarding mental health disorders, the ability to recognise warning signs, knowledge of help-seeking options, and skills to support others who are developing a mental health disorder [13]. Mental health literacy is generally poor amongst adolescent males and is a barrier to help-seeking for mental health problems [14]. Poor mental health literacy as indicated by poor symptom recognition and high levels of stigma may also be a barrier to seeking help among athletes [15], and could be an important component of sports programmes going forward [16]. In sport, psychological resilience is defined as the mental processes (e.g., problem solving) and behaviours (e.g., accessing social support) which promote personal assets and protect an individual from the potential negative effect of stressors [17]. For example, psychological resilience can provide a buffer to the onset of mental health problems following common stressors such as injuries among young athletes [18]. Indeed a combination of psychological resilience and mental health literacy may be key to facilitating the most optimal mental health outcomes among young athletes when they face significant stressors [18]. Both constructs lend themselves to intervention, however, studies in this area have been of low quality and more robust interventions, using stronger research designs are needed to inform policy and guide principles of best practice in mental health care in organised sports [11]. 
One way of increasing the quality of interventions is to intervene at multiple levels within a single setting. Such approaches are based on the socio-ecological approach to health promotion [19]. Models of sports-based health promotion advocate for multi-level interventions due to the multiple, interdependent levels of influence that operate on one's health behaviours within the sport setting - including intra- and interpersonal levels of influence, as well as influence from sports club administrators and the sports club setting itself [20, 21]. Interventions that have demonstrable efficacy, and that may be considered best practice in sports-based mental health promotion have been multilevel in nature (e.g., intervening with athletes, coaches, parents[22]). Such interventions offer an evidence- and theory-based approach to mental health promotion in the organised sports setting.

The purpose of this study was to test the effectiveness of a sports-based program designed to promote early mental health intervention and help seeking for existing and developing mental health disorders, as well as psychological resilience among adolescent male sport participants (Ahead of the Game; AOTG). Using a community-based and communitymatched, non-randomised controlled design, adolescent male sports participants drawn from participating community sporting clubs were offered a multi-component (e.g., mental health literacy, resilience), multi-level (e.g., programs for adolescents, coaches, and parents) program through their sport club. In this paper we report the results pertaining to athlete-level data while coach and parent data are reported elsewhere. We hypothesised that, compared with adolescents from sports clubs in a control community, adolescents who participated in the intervention would show increases in the primary outcomes of mental health literacy, intentions to seek and provide help, resilience, and a decrease in stigmatising attitudes [23]. We also hypothesised increases 
among athletes in the intervention group for the secondary outcomes of perceived familial support and implicit beliefs regarding one's ability to deal with problems when compared to athletes in the control group.

\section{METHOD}

\section{Study Design}

The Community-Based Participatory Research (CBPR) framework [24] served as the conceptual framework for the development and evaluation of the AOTG intervention. CBPR is a collaborative approach to research that equitably involves all partners in the research process and recognises the unique strengths that each brings. Our community engagement in the design and development of the AOTG program has been reported elsewhere [25-27]. In line with the CBPR framework, the multi-level, multi-component AOTG program was tested at a community level. We purposively selected the intervention community in Eastern Australia based on the presence of urban and rural settings, club accessibility, and the proximity to the institution responsible for implementing the program. We chose a matched control community (another urban and regional area in Eastern Australia) based on its similarity to the intervention community in terms of location, size, socioeconomic status, and number of sporting clubs. In reporting this study, we have followed the Template for Intervention Description and Replication (TIDIER) checklist [28], and where applicable, the Consolidated Standards of Reporting Trials (CONSORT) statement [29]. 


\section{Participants}

Adolescent males, aged 12 - 17 years, who participated in community-based non-elite organised sports clubs were recruited to an intervention group or community-matched control group. Parents/caregivers and coaches of adolescent participants were also recruited to the intervention group. All sports and clubs were eligible; however, the study was conducted during winter 2017 when the primary participatory sports in Australia for adolescent males are soccer, Australian Rules football, rugby league, and basketball. Other sports operating during this time include swimming and rugby union. Eligible teams included those competing in the 'Under 13' through to 'Under 18' age groups.

\section{Procedure}

The study protocol was registered in the Australian New Zealand Clinical Trials Registry (ACTRN12617000709347). Ethics approval was obtained from the Institutional Human Research Ethics Committee. All adolescent participants were recruited through participating sports clubs. Clubs were notified about the program prior to the start of the sporting season and invited to participate (via email or letter directed to the club administrator) by the research team. Clubs were offered presentations about the program on an as-needed basis. Individual adolescent participants were contacted through their team and/or through club generated notices via email or social media. Written informed consent was obtained from all participants and parental consent was obtained for all participants aged 15 years and younger. Subsequently, parents/caregivers and coaches of those adolescent participants were also invited to participate in the study. Following the consent process, baseline measures were completed, and the first intervention component was scheduled. The questionnaire consisted of 135 items and took approximately 30 
minutes to complete. Measures were completed using paper and pencil at the training ground or clubhouse of participating teams (intervention group), or at home and returned in a sealed envelope (control group). Follow-up measures were taken one month following completion of the program components (and approximately 6-8 weeks following baseline measures) in the same way and location as the baseline measures. Data were collected at the sport club's training ground or at the local University campus. Participants were recruited between February and July 2017, and workshops were delivered between May and August 2017. We completed participant recruitment and workshop delivery in order to complete the study prior to end of the winter sporting season in Australia (September 2017). Strategies to improve all workshops and data collection processes, and to maximise fidelity of delivery and dose delivered are reported in detail elsewhere [30].

\section{Ahead of the Game Program (Intervention)}

The overall program and each intervention component are described in detail within the study protocol [23]. Adolescents were invited to take part in a brief team-based, face-to-face mental health literacy workshop named "Help Out a Mate". The Help Out a Mate workshop was usually delivered after a routine scheduled practice and took approximately 45 minutes. This component focused on helping adolescents to recognise the signs of depression and anxiety, encourage help-seeking and help-provision, make an adult aware of potential mental health problems, and understand self-help behaviours. Additionally, this component addressed the issue of how to ask for help if an adolescent felt that he needed it. Additional resources provided to adolescents included a business card which listed key steps on how to help a friend and provided a list of local mental health resources (based on concept testing from focus groups, we called this 
a 'Man Card' for the purposes of the intervention to counteract the stigma young men feel when talking about mental health). All workshops were delivered by a member of the research team and a volunteer who was accredited with basic mental health training and received training to deliver the Help Out a Mate workshop. Workshops were delivered at the club's training ground, or the local University campus.

Adolescents were also invited to take part in an internet-supported resilience intervention named "Your Path to Success in Sport". The intervention targeted key psychological skills identified in sport-based resilience literature (e.g., [31]), and aimed to help adolescent males cope with adversity through explicit sport-based examples. This component was delivered via a brief, team-based, face-to-face workshop (approx. $45 \mathrm{~min}$ ) supported by six internet-based (website/mobile application) modules lasting approximately 15 minutes each. The workshop was framed around expectations versus reality in the process of achieving one's goals (i.e., the "path to success") to identify inevitable adversities that adolescents are likely to face in and outside of sport. The workshop also allowed participants to sign up to the internet-based modules, and to complete the first module within the session, to enhance subsequent uptake and engagement. All workshops were delivered by a member of the research team or a registered sport psychology practitioner employed as a research assistant. The internet-based modules addressed: (i) problemsolving; (ii) controlling the controllables; (iii) managing your thoughts; (iv) keeping your cool; (v) playing to your strengths; and (vi) appreciating your team. An additional recap module was also available to participants who completed all six preceding modules. Workshops were again delivered at the club's training ground or on the local University campus. 
The parent component was designed to increase parent mental health literacy regarding adolescent mental health via a one-hour, face-to-face workshop. Specifically, the workshop aimed to raise parents' awareness of their role in promoting and supporting positive adolescent mental health, and to increase knowledge of common adolescent mental health disorders, positive mental health-promoting behaviours, and help-seeking options. This workshop also aimed to reduce stigma, promote constructive communication about mental health, and increase parental confidence to support adolescent mental health. The parent component was delivered at a club level, rather than a team level (i.e., parents from multiple teams in each club attended the same session). All workshops were delivered by a member of the research team at the local University campus or at the sports club where facilities allowed.

Based on Keyes' mental health continuum model [32], the purpose of the coach education component was to facilitate higher levels of wellbeing among athlete participants by complimenting the focus on prevention and resilience regarding mental health problems in the other components. The coach education component was based on self-determination theory principles with content drawn largely from an intervention shown previously to decrease player burnout across a season [33]. Self-determined motivation among young athletes has been shown to be negatively associated with levels of psychological distress and indicators of mental health problems, and positively associated with wellbeing and mental health [34-37]. The coach intervention was designed to give coaches strategies to support their players' basic psychological needs, including autonomy (feeling self-directed and capable of making choices about one's actions), competence (feeling effective in one's interactions with the physical and social environment), and relatedness (feeling closely connected and cared for by others). This 
component used a blended delivery model, including two face-to-face workshops and two mentoring sessions delivered by a registered sport psychology practitioner, plus 11 self-paced online modules. All workshops were delivered on the local University campus in rooms fitted with a projector.

Lastly, the supplementary messaging campaign was based on a 'Man Card' concept (e.g., "You won’t lose your Man Card for getting help"). The concept used a combination of humour, credible and relatable images, as well as relevant and positive mental health messages. Materials included posters and banners that were placed in visible areas around each club, as well as branded merchandise for adolescents and a campaign-specific website.

\section{Control Group}

Participating clubs in the control group received usual practice (i.e., their standard sport programming without any intervention from the research team). All clubs were offered a mental health literacy program upon completion of the study. Data were provided by adolescents within each club, as well as their parents/caregivers, and coaches. Only adolescent data are reported here.

\section{Outcome Measures}

Depression and Anxiety Literacy. Thirteen items of the Depression Literacy Questionnaire [38] relevant to the AOTG program were used to measure depression literacy. Similarly, 13 items from the Anxiety Literacy Questionnaire were used to measure anxiety literacy [39]. For both questionnaires, each item presents a statement about depression and respondents are asked to 
choose one from one of three options for each statement (true, false, or I don't know). Correct answers are scored with one point, while incorrect answers and responses of 'I don't know' are scored with a zero. The total score for each scale represents that total number of correct answers with a maximum score of 13. Example items include 'Sleeping too much or too little may be a sign of depression' and 'People with anxiety disorder often hear voices that are not there'. Both the Depression Literacy Questionnaire $(\alpha=.78)$ [38], and the Anxiety Literacy Questionnaire ( $\alpha$ =.76) [39] have acceptable internal consistency.

Resilience. The 10-item version of the Connor-Davison Resilience Scale [40] was used to measure resilience. Respondents are asked to indicate how much they agree with a set of statements as they apply to them over the last month. All items are rated on a Likert scale from 1 (not true at all) to 5 (true nearly all of the time). A total score is computed by summing the score on each item. An example item is 'I am not easily discouraged by failure'. The scale has good internal consistency $(\alpha=.85)$ [40]. Baseline internal consistency in the current study was excellent $(\alpha=.88)$.

Confidence to seek help. A single item drawn from the Mental Health Literacy Scale [41] -'I am confident that I know where to seek information about mental illness'- was used to measure confidence to seek information regarding mental illness. The item is scored on a Likert scale from 1 (strongly disagree) to 5 (strongly agree).

Help seeking intentions. The General Help Seeking Questionnaire (GHSQ) [42] is a flexible measure developed to assess help seeking intentions across a variety of contexts. The GHSQ was 
adapted for the sport context (i.e., by adding coach as an item) and used to measure intentions to seek help from formal and informal sources. Eleven items were used to assess intentions to seek help from formal (e.g., doctor) and informal sources (e.g., friend, parent, coach). Each item asks 'If you were having a personal or emotional problem, how likely is it that you would seek help from the following people?' and is scored on a Likert scale from 1 (extremely unlikely) to 7 (extremely likely), with a 'not applicable' option also available. Mean scores were calculated separately for formal and informal sources. Among adolescents, the GHSQ has adequate internal consistency $(\alpha=.70)$ [42]. In the current study, the internal consistency of the GHSQ at baseline among all participants was good $(\alpha=.83)$.

Stigmatising Attitudes. The youth version of the Social Distance Scale [43] was used to measure stigmatising attitudes, adapted for the current study. Five items were used to measure the respondents' self-reported willingness to have contact with a person with mental illness. Items were rated on a Likert scale from 1 (definitely unwilling) to 5 (definitely willing). An example item is 'How willing would you be to work on a project with someone with a mental illness?' Internal consistency of the Social Distance Scale at baseline among all participants in the current study was excellent $(\alpha=.91)$.

Implicit beliefs. Implicit beliefs regarding one's ability to deal with problems were measured using three items adapted from previous research [44]. The items include: 'You have a certain way of dealing with problems and you really can't do much to change it'; 'You have very little control over the way that you deal with problems'; and, 'Some people are just naturally better at dealing with problems than others'. All items were scored on a Likert scale from 1 (strongly 
disagree) to 5 (strongly agree). Mean item score was calculated, with lower scores indicating more adaptive beliefs. Internal consistency of the three items at baseline and among all participants was low $(\alpha=.47)$.

Perceived familial support. Perceived familial social support was measured using the four items of the parental support subscale of Multidimensional Scale of Perceived Social Support [45]. An example item is: 'I get the emotional help and support I need from my family'. All items were scored on a 7-point Likert scale from 1 (very strongly disagree) to 7 (very strongly agree). Mean item scores were calculated from all four items. Internal consistency for the four items among all participants at baseline was excellent $(\alpha=.91)$.

Psychological distress. Psychological distress was measured using the Kessler-6 (K6) scale [46]. The K6 is six-item screening tool that assesses the level of depressive and anxiety symptoms experienced within the past month. The K6 is scored on a 5-point Likert scale from 0 (none of the time) to 4 (all of the time). All items contain the stem 'During the past 30 days, about how often did you feel'. An example item is: 'hopeless?' The K6 has good internal reliability among a sample of adolescents $(\alpha=.84)$, and among adolescent males $(\alpha=.83)$ [46]. Internal consistency at baseline in this study was adequate $(\alpha=.74)$.

Wellbeing. Wellbeing was measured using the 14-item version of Keyes' Mental Health Continuum adapted for adolescents (MHC) [47]. The MHC is a measure of an individual's emotional, psychological and social wellbeing. The short form of the MHC has shown excellent internal consistency $(\alpha>.80)$ and discriminant validity in nationally representative samples of 
adolescents [47]. All items were summed to compute a total score. Internal consistency among all participants at baseline in this study was excellent $(\alpha=.92)$.

Covariates. Neighbourhood level socio-economic position was determined according to the Socio-Economic Indexes for Areas Index of Relative Socio-Economic Disadvantage (SEIFA) [48] using participants' home postcodes. Participants self-reported their age, indigenous status, and language spoken at home.

\section{Required Sample Size}

Rather than power on outcomes which were specific only to some program components (e.g., mental health literacy or resilience), we calculated the required sample size using the general indicator of psychological distress (K6). Based on pilot testing using two independent samples with a between group difference of $1.2(\mathrm{SD}=4.0)$, adjusting for clustering using a design effect of 1.35 we required 231 participants in each group (with an alpha of 0.05 and 80\% power).

\section{Statistical Analyses}

Independent samples $t$-tests were used to examine baseline differences between the intervention and control groups, as well as differences between participants who completed the program per-protocol and those who did not. Between-group mean differences on the primary and secondary outcomes were analysed using linear mixed models (LMM) in SPSS Version 25.0. Group (intervention vs control), time (baseline and one month follow up), and the group by time interaction, were included as fixed factors in the model. Participant age and socioeconomic 
status were entered as covariates. All models adjusted for clustering at the team and club levels.

The linear mixed model uses all available data and, therefore, cases with missing values are included in the analysis. This model uses restricted maximum likelihood for parameter estimation from all available cases regardless of whether or not they contain missing data. Effectiveness of the AOTG program in increasing all primary and secondary outcomes was established via a change from baseline to post-intervention in the intervention group relative to the control group, reflected in the group by time interaction at $P<.05$ level.

All participants were included in intention-to-treat analyses. We also conducted an exploratory per protocol analysis in which we compared only those in the intervention group who had completed the program per protocol with all participants in the control group. For this analysis, we defined per protocol as attendance at the Help Out a Mate workshop, attendance at the Your Path to Success in Sport workshop, completion of the six online modules, and having at least one parent who attended the parent program. Given the low number of coaches completing the program, we did not include a per protocol criterion for this component in the per protocol analysis.

Changes from Trial Registration. Based on piloting and formative evaluation, some changes to the method reported in the trial registration were made. First, due to lack of uptake, the coach program was shortened from an 11-module program (delivered over three months) to a 6-module program (delivered in up to four weeks). In line with this, the follow up period was also shortened from 12 weeks from baseline to 6-8 weeks from baseline. More refined measures of anxiety and depression literacy were used, replacing the Mental Health Literacy Scale [41] 
because the items of the new scales provided greater overlap with intervention content and therfeore a better measure of any potential intervention effect. One item from the Mental Health Literacy Scale [41] was retained to measure confidence to seek mental health information. Other measures related to athlete motivation, engagement and burnout, as well as parent- and coachlevel data were collected in line with the study protocol [23] and will be reported separately. Similarly, the qualitative components of the evaluation will also reported separately [e.g., 49]. Finally, the sample size calculations reported here were updated from the trial registration based on data captured during formative evaluation. Calculations were updated prior to data being collected in the current study.

\section{RESULTS}

\section{Descriptives.}

Participants and their recruitment. Initial contact was made with 28 sports clubs to participate in the intervention. Eighteen of those clubs expressed an interest in participating in the program, and formal letters of support to participate were received from nine clubs. One club who provided a letter of support to participate did not end up participating due to time constraints. In total, there were 22 distinct team units from eight clubs recruited to the intervention group. A total of 350 adolescent males were recruited with a mean age of 14.53 years ( $S D=1.19$ years). Most clubs $(n=6 / 8 ; 75 \%)$ were soccer clubs, with one rugby league club $\left(n_{\text {participants }}=16 ; 7 \%\right)$ and one swimming club $\left(n_{\text {participants }}=11 ; 4.8 \%\right)$ also participating. The number of participants per team ranged from 8 to 20 , with a mean of $13(S D=3.53)$. Seven $(2 \%)$ participants spoke a language other than English at home, and four (1.1\%) identified as Aboriginal or Torres Strait Islander. 
Thirty-one clubs were invited to take part from the control community. A total of 14 clubs participated, which included 11 soccer clubs, two Australian football clubs, and one basketball club. A total of 466 participants were drawn from the 14 clubs over a total of 72 distinct teams. The number of participants per team ranged from 1 to 23, with a mean of 6.43 $(S D=4.76)$. The mean age of participants in the control group was 14.66 years $(S D=1.39$ years). Ten $(2.1 \%)$ participants spoke a language other than English at home, and $16(3.1 \%)$ identified as Aboriginal or Torres Strait Islander.

In the intervention group, 180 parents, with a mean age of 47.70 years $(S D=5.14$ years), took part in the parent workshop across all eight intervention clubs. Eight coaches $\left(M_{\mathrm{age}}=45.37\right.$ years, $S D=10.58$ years) also took part in the coach education component. All coaches attended the first workshop and completed the first online module; however, only one coach completed all modules.

\section{Participation numbers in the intervention.}

Adolescents. In total, $283(80.9 \%)$ adolescent participants attended at least one program.

Of those, $271(95.8 \%)$ attended the Help Out a Mate workshop, and $251(88.7 \%)$ attended the Your Path to Success in Sport workshop. Of these, 91 (32.2\%) completed all six online modules, with an average of $2.46(S D=2.96)$ online modules completed across all participants in the intervention group. A total of $85(30.0 \%)$ participants completed all adolescent components of AOTG. 
Parents and coaches. Parents or caregivers of 162 (57.2\%) intervention participants attended parent workshops. A total of eight coaches attended the first coach workshop, representing a total of $57(19.3 \%)$ adolescents.

Adolescent per protocol. The number of adolescent participants who completed all adolescent components and who had a parent attend a parent workshop was 45 (15.9\%; in the per-protocol analyses these participants were considered to have completed the intervention).

\section{Baseline characteristics.}

Estimated marginal means and 95\% confidence intervals of the intervention and control groups across all study variables at both time points are presented in Table 1. The baseline characteristics for all study variables of those participants in the intervention group who completed the program per protocol and those who did not are given in Table 2, including tests of baseline differences between the groups.

Intervention effects: Intention-to-Treat. Estimated marginal means and 95\% confidence intervals by group and time, as well as significance values for all group by time interactions for all outcome variables are given in Table 1. Significant group by time interactions were present for the primary outcomes of depression literacy $\left(F_{1,466.68}=36.94, p<.001\right)$ and anxiety literacy $\left(F_{1,457.39}=46.54, p<.001\right)$, as well as intentions to seek help from formal sources $\left(F_{1,467.55}=\right.$ 7.17, $p=.008)$, confidence to seek mental health information $\left(F_{1,450.31}=5.00, p=.026\right)$, and resilience $\left(F_{1,423.77}=10.71, p=.001\right)$. There was also a significant group by time interaction for the secondary outcome of wellbeing $\left(F_{1,425.87}=10.73, p=.001\right)$. For all outcomes where a 
significant group by time interaction was found, adolescents in the intervention group reported significantly greater increases at follow up compared with adolescents in the control group. There were no significant group by time interactions for social distance (stigma), intentions to seek help from informal sources, implicit beliefs, perceived familial support, or psychological distress. No negative effects of the intervention were reported.

Intervention effects: Per-Protocol. Estimated marginal means and 95\% confidence intervals by group (those who completed the intervention per protocol, and those in the intervention group who completed less than per protocol) and time, as well as significance values for all group by time interactions for all outcome variables are given in Table 3 . The pattern of results for the perprotocol analyses largely mirrored those of the intention-to-treat analyses. However, those who completed the AOTG program per protocol reported decreases in social distance $\left(F_{1,264.98}=6.51\right.$, $p=.011)$ and implicit beliefs $\left(F_{1,299.24}=13.77, p<.001\right)$, both of which represent more adaptive outcomes following the intervention. Further, there was no group by time interaction for confidence to seek mental health information $\left(F_{1,274.73}=0.178, p=.673\right)$ or intentions to seek help from formal sources $\left(F_{1,264.98}=6.51, p=.196\right)$ for per-protocol analyses, whereas positive intervention effects were evident for these outcomes in the intention to treat analyses.

\section{DISCUSSION}

Using a non-randomised, community-matched design, we tested the effectiveness of a sports-based program designed to promote early mental health intervention and help seeking for existing and developing mental health disorders, as well as psychological resilience among adolescent male sport participants. As hypothesised, the AOTG intervention showed significant 
benefits on depression literacy, anxiety literacy, intentions to seek help from formal sources, confidence to seek mental health information, resilience, and wellbeing. No significant effects were found for social distance (stigma), intentions to seek help from informal sources, implicit beliefs, perceived familial support, or psychological distress. No negative effects were evident for psychological distress. Results were similar for those participants who completed the program per protocol when compared with those who did not. As such, the AOTG program was largely successful in achieving its key aims and equipping young men who participate in organised sports with the knowledge, intentions, confidence, and psychosocial resources necessary to promote mental health and support better identification of, and response to mental health problems.

The current study is the first to provide evidence that a mental health intervention in organised sport can be effective for a sample of non-elite, adolescent male sport participants. A recent systematic review of sports-based mental health interventions has shown that a range of positive mental health outcomes can be facilitated through interventions [11]. However, the quality of the 10 studies in that review was generally poor with small sample sizes and a lack of control group the most common problems [11]. In a non-controlled intervention study among selected players, parents, and coaches within junior Australian football clubs, brief mental health literacy training was associated with increases in knowledge of mental health disorders, confidence in helping someone with a mental health disorder, and more positive attitudes toward people with mental illness [50]. Our study extends these findings in a similar context using a similar spread of participants, and with the additional strength of causal attributions owing to the controlled nature of the current study. Similarly, participation in a 12-hour mental health first aid 
program by club leaders of Australian football clubs in rural Australia showed some associated increases in leaders' ability to recognise mental health disorders and increased confidence to respond to mental health difficulties in others [51]. However, the benefits of training club leaders didn't filter down to the athlete level where no attitudinal change was evident. The key difference between these programs and the AOTG program was intervention at multiple levels, most notably among athletes.

Several key features of the AOTG program may explain its effectiveness. To our knowledge, this is the first sport-based intervention to combine both resilience-focused and mental health literacy-focused programming. Consistent with Keyes model of mental health [32], this combination may have a cumulative effect in the promotion of one's current mental health and wellbeing. Specifically, cumulative benefits may be derived by equipping participants with the skills to recognise the warning signs of mental health problems (mental health literacy), as well as the skills to deal with any problems that they may face in the future (resilience). Second, the blended delivery model used in the current study (face-to-face workshops supplemented with online modules) was also likely to be beneficial. The combination of face-to-face and online components has been recommended within interventions for adolescents [52] and may be more effective than face-to-face delivery alone [53].

Third, we adopted a CBPR framework [24] for the design and implementation of the AOTG program [30]. This included a large amount of formative research with end-users to ensure that their needs were met regarding the content and delivery of the program [25-27]. Community end-users within Australian community sporting clubs have emphasised that the 
implementation of programs within such clubs should be planned to reflect the real-world context within which the clubs operate. This includes the volunteer-driven nature of community sports clubs and the subsequent resource challenges which include human resources, operational difficulties, and financial burdens [54]. Given the time constraints of those working in organised sports $[25,26]$ the combination of resilience and mental health literacy components delivered via a blended model and designed in conjunction with end users may be ideally suited to sports contexts to maximise engagement, feasibility, and effectiveness. The limitation to the CBPR approach is that the generalisability of the intervention is unknown because the design and implementation of the intervention is dictated by the community-based end users. It is therefore unclear how acceptable and engaging the concepts employed in this study such as a 'Man Card' would be when deployed outside of the defined community.

Given the brief nature of the program, it is unsurprising that those who completed the program per protocol reported some extra benefit - decreases in stigmatising attitudes, and more adaptive implicit beliefs regarding one's ability to deal with problems. In contrast, there was no effect among these participants for intentions to seek help from formal sources or confidence to seek mental health information. This may be due to ceiling effects being present at baseline in this subsample of high adherents (mean of 4.65 out of 5 for formal help seeking, and 4.07/5 for confidence to seek information). Differences in intentions to seek help may also be explained by participants who completed more of the protocol feeling more capable of managing mental health problems on their own, particularly where the resilience program was completed in full [55]. This may also be true for those whose parents also participated because they may be more sensitive to the needs of their adolescent and may provide additional support, reducing the 
perceived need to seek help [56]. Although multi-component programs are promising in this area [55], they require investments of time from multiple people. It is important to find a balance between feasibility and effectiveness whereby positive outcomes are maximised through the optimal combination of the two.

The AOTG program was implemented at multiple levels of influence, in line with the settings approach to sports-based health promotion [20] and the sports setting matrix for implementation of community sports-based interventions [21]. This is in contrast to previous non-controlled studies within junior sporting clubs that have used similar content to intervene only at the administrator and club leadership level $[50,51]$. The settings approach specifies three distinct levels of influence which each have reciprocal relationships with the individual. These levels include the micro level (e.g., actions of practitioners such as coaches), meso level (e.g., actions of club administrators), and macro level (e.g., club policies) [20]. According to this model, the AOTG program was aimed at the micro level (e.g., intra- and inter-personal influences) and the meso level (i.e., club-based promotional campaign). Similarly, the implementation matrix for community sports specifies a number of levels of influence including the individual, team, club, regional association, and governing bodies [21]. According to this model, the AOTG program was implemented at the individual, team (i.e., team level delivery of intervention components), and club levels. To the extent that the AOTG intervention was successful, this may have been due to the multi-level nature of the intervention which is likely to enhance the effectiveness of sports-based health interventions. However, greater change may be facilitated by intervention at all levels of influence. For example, a similar mental health literacy 
program run in similar Australian junior sports contexts showed no benefit at the athlete level when intervening with club leaders alone [51].

No effect of the intervention - across intention to treat or per protocol analyses - was found for intentions to seek help from informal sources, perceived familial support, or psychological distress. Intentions to seek help from informal sources such as friends was relatively high at baseline among both groups $(4.58 / 5$ for the intervention group and $4.66 / 5$ for the control group, respectively) which may limit any potential effect of the intervention due to ceiling effects. This may be a typical pattern Australian adolescents [42]. It may also be that selfselection biases meant that parents who already provided high levels of support were more likely to attend the parent program and thereby also limit any potential effect of the intervention. For example, those adolescents who completed the program per protocol had higher levels of familial support at baseline when compared to those adolescents who did not complete the program (see Table 2). This is typical of family-level interventions [57], particularly where fathers are involved [58]. Finally, the reduction of psychological distress may not be a short term outcome of this program given the aim of the program to promote early intervention, help-seeking, and resilience. It is possible that reduction in psychological distress may be evident over time as a result of increases in wellbeing and resilience; however, we did not measure longer term outcomes. It is also noteworthy that no increase in psychological distress was evident as a result of the program. These results are consistent with a classroom-based mental health literacy programs for adolescents [59]. 
Given the typically low quality of mental health interventions in sport [11], this study has some important strengths - most notably, the large sample size and controlled nature of the study. However, the community-matched control design increases the risk of bias from potential confounding effects such as local media coverage on mental health or school-based initiatives being run within the selected communities. Furthermore, we have not accounted for the effect of variations in implementation of the AOTG program, which may have a meaningful effect on program outcomes and recommendations for scalability of the program to a broader context. Our design did not allow for us to assess which component of the intervention may have the highest leverage. Future research testing stand-alone components of the program would allow for researchers to identify the active ingredients of the program. Finally, the one month postintervention follow-up period does not give an indication as to the long-term outcomes of the program, and increases the likelihood of positive outcomes when compared with longer followup periods. Randomised controlled trials with longer follow-up periods would give a stronger indication as to the sustained effects of the program and its components on all outcomes including help-seeking behaviours and long-term mental health outcomes. Finally, our measure of implicit beliefs about one's ability to handle adversity in life had poor internal consistency, and results pertaining to this item should be interpreted with some caution.

In this study we tested the effectiveness of a multi-component sports-based program to promote early mental health intervention and help seeking for existing and developing mental health disorders, as well as psychological resilience among adolescent male sport participants. Results demonstrate that the program was largely successful, with positive and significant outcomes for participants who received the AOTG program. Given high rates of sport 
participation worldwide [6] and the engaging nature of organised sports that lends itself to mental health intervention [27], translation and dissemination of the AOTG program to other youth populations and communities may be beneficial. With over $40 \%$ of adolescents participating in sport around the world [6], there is meaningful potential to improve the mental health of adolescent males globally through sports-based intervention. However, given the community-based design used in this study, additional formative research to support translation and dissemination of the program to other communities is necessary. Nevertheless, multicomponent, multi-level, blended interventions may provide avenues for promoting resilience amongst adolescent males in a way that is both effective and scalable. 
Acknowledgements: We acknowledge the valuable input of Professor Sandra C. Jones into the conception, design, and evaluation of the study. We are grateful for her time and expertise in making a substantial contribution to the study. We are also grateful for the time and effort of all participants, and the sporting organisations involved in the study for their support. Results of this study do not constitute endorsement by the American College of Sports Medicine.

Conflict of interest: Dr Vella and Dr Swann currently consult to the Movember Foundation on related projects.

Sources of Funding: The Ahead of the Game study was funded by the Movember Foundation through an Australian Mental Health Initiative grant. The project also received funding from the University of Wollongong Global Challenges Program. 


\section{REFERENCES}

1. Costello EJ, Egger H, Angold A. 10-year research update review: the epidemiology of child and adolescent psychiatric disorders: I. Methods and public health burden. J Am Acad Child Adolesc Psychiatry. 2005;44:972-86.

2. Kessler RC, Berglund P, Demler O, Jin R, Merikangas KR, Walters EE. Lifetime prevalence and age-of-onset distributions of DSM-IV disorders in the National Comorbidity Survey replication. Arch Gen Psychiatry. 2005;62:593-602.

3. Merikangas KR, He J-P, Burstein M et al. Lifetime prevalence of mental disorders in US adolescents: results form the National Comorbidity Study-Adolescent Supplement. J Am Acad Child Adolesc Psychiatry. 2010;49:980-9.

4. Seidler ZE, Dawes AJ, Rice SM, Oliffe JL, Dhillon HM. The role of masculinity in men's help-seeking for depression: a systematic review. Clin Psychol Rev. 2016;49:106-18.

5. Griffith DM. Biospsychosocial approaches to men's health disparities research and policy. Behav Med. 2016;42:211-5.

6. Aubert S, Barnes JD, Abdeta C et al. Global Matrix 3.0 Physical Activity Report Card Grades for Children and Youth: results and analysis from 49 countries. J Phys Act Health. 2018;15(Suppl 2):S251-S73.

7. Australian Bureau of Statistics. Children's participation in sport and leisure time activities 2003-2012 [Internet]. Canberra, Australia. 2012 [cited 2020 Jun 06]. Available from: https://www.ausstats.abs.gov.au/ausstats/subscriber.nsf/0/ABA491F2AC01415FCA257A DA000E3EAA/\$File/4901055001_2003\%20-\%202012.pdf 
8. Vella SA, Cliff DP, Okely AD, Scully M, Morley B. Associations between organized sports participation and obesity-related health behaviors in Australian adolescents. Int $\mathbf{J}$ Behav Nutr Phys Activ. 2013;10:113.

9. Baker D, Rice S. Keeping it real: reimagining mental health care for all young men [Internet]. Melbourne, Australia: Orygen, The National Centre of Excellence in Youth Mental Health. 2017 [cited 2020 Jun 06]. Available from: https://www.orygen.org.au/Policy/Policy-Reports/Keeping-it-real/Keeping-it-real-PolicyPaper?ext=.

10. Liddle SK, Deane F, Vella SA. Addressing mental health through sport: a review of sporting organizations' websites. Early Interv Psychiatry. 2017;11:93-103.

11. Breslin G, Shannon S, Haughey T, Donnelly P, Leavey G. A systematic review of interventions to increase awareness of mental health and well-being in athletes, coaches and officials. Syst Rev. 2017;6(1):177.

12. Ho FKW, Louie LHT, Wong WH, Chan KL, Tiwari A, Chow CB et al. A sports-based youth development program, teen mental health, and physical fitness: an RCT. Pediatrics. 2017;140:e20171543.

13. Jorm AF. Mental health literacy: empowering the community to take action for better mental health. Am Psychol. 2012;67:231-43.

14. Haavik L, Joa I, Hatloy K, Stain HJ, Langeveld J. Help seeking for mental health problems in an adolescent population: the effect of gender. J Mental Health. 2019;28:46774. 
15. Reardon CL, Hainline B, Miller AA, Baron D, Baum AL, Bindra A et al. Mental health in elite athletes: International Olympic Committee consensus statement (2019). Brit J Sports Med. 2019;53:667-99.

16. Breslin G, Smith A, Donohue B, Donnelly P, Shannon S, Haughey TJ et al. International consensus statement on the psychosocial and policy-related approaches to mental health awareness programmes in sport. BMJ Open Sport Exerc Med. 2019;5:e000585.

17. Fletcher D, Sarkar M. Psychological resilience: A review and critique of definitions, concepts, and theory. Eur Psychol. 2013;18:12-23.

18. Putukian M. The psychological response to injury in student athletes: a narrative review with a focus on mental health. Brit J Sports Med. 2016;50:145-8.

19. Sallis JF, Owen N. Ecological models of health behavior. In: Glanz K, Rimer BK, Viswanathan M, editors. Health Behavior: Theory, research, and practice. San Francisco, CA, USA: Jossay-Bass; 2015. p. 43-64.

20. Kokko S. Sports clubs as settings for health promotion: fundamentals and an overview of research. Scand J Public Health. 2014;42(Supp. 15):60-5.

21. Finch CF, Donaldson A. A sports setting matrix for understanding the implementation context for community sport. Br J Sports Med. 2010;44:973-8.

22. Martinsen M, Bahr R, Borresen R, Holme I, Pensgaard AM, Sundgot-Borgen J. Preventing eating disorders among young elite athletes: a radomised controlled trial. Med Sci Sports Exerc. 2014;46:435-47. 
23. Vella SA, Swann C, Batterham M et al. Ahead of the Game Protocol: A multi-component, community sport-based program targeting prevention, promotion and early intervention for mental health among adolescent males. BMC Public Health. 2018;18:390. DOI:10.1186/s12889-018-5319-7

24. Minkler M, Wallerstein N. Community-based Participatory Research for Health. $2^{\text {nd }}$ ed. San Francisco: Jossey-Bass; 2008. 544 p.

25. Ferguson H, Swann C, Liddle SK, Vella SA. Investigating youth sports coaches' perceptions of their role in adolescent mental health. J Appl Sport Psychol. 2018;31:235252.

26. Hurley D, Swann C, Allen MS, Vella SA. The role of community sports clubs in adolescent mental health: the perspectives of adolescent males' parents. Qual Res Sport Exerc Health. 2017;9:372-88.

27. Swann C, Telenta J, Draper G et al. Youth sport as a context for supporting mental health: adolescent male perspectives. Psychol Sport Exerc. 2018;35:55-64.

28. Hoffmann T, Glasziou P, Boutron I et al. Better reporting of interventions: template for intervention description and replication (TIDieR) checklist and guide. BMJ. 2014(248):g1687. DOI: 10.1136/bmj.g1687

29. Moher D, Hopewell S, Schulz KF et al. CONSORT 2010 explanation and elaboration: updated guidelines for reporting parallel group randomised trials. BMJ. 2010;340:c869. DOI: $10.1136 /$ bmj.c869 
30. Vella SA, Swann C, Boydell KM et al. Sports-based mental health promotion in Australia: formative evaluation. Psychol Sport Exerc. DOI: 10.1016/j.psychsport.2019.101560

31. Fletcher D, Sarkar M. A grounded theory of psychological resilience in Olympic champions. Psychol Sport Exerc. 2012;13(5):669-78.

32. Keyes CL. The mental health continuum: from languishing to flourishing. J Health Soc Res. 2002;43:207-22.

33. Langan E, Toner J, Blake C, Lonsdale C. Testing the effects of a Self-Determination Theory-based intervention with youth Gaelic football coaches on athlete motivation and burnout. Sport Psychol. 2015;29(4):293-301.

34. Stenling A, Lindwall M, Hassmén P. Changes in perceived autonomy support, need satisfaction, motivation, and well-being in young elite athletes. Sport Exerc Perform. 2015;4:50-61.

35. Sheehan RB, Herring MP, Campbell MJ. Associations between motivation and mental health in sport: a test of the hierarchical model of intrinsic and extrinsic motivation. Front Psycho. 2018;9(707). doi: 10.3389/fpsyg.2018.00707.

36. Vella SA, Benson A, Sutcliffe J, McLaren C, Swann C, Schweickle MJ et al. Selfdetermined motivation, social identification and the mental health of adolescent male team sport participants. J Appl Sport Psychol. 2020. doi: doi.org/10.1080/10413200.2019.1705432 
37. White RL, Parker PD, Lubans DR, MacMillan F, Olson R, Astell-Burt T et al. Domainspecific physical activity and affective wellbeing among adolescents: an observational study of the moderating roles of autonomous and controlled motivation. Int J Behav Nutr Phys Act. 2018;15:87.

38. Griffiths KM, Christensen H, Jorm AF, Evans K, Groves C. Effect of web-based depression literacy and cognitive-behavioural therapy interventions on stigmatising attitudes to depression: Randomised controlled trial. Br J Psychiatry. 2004;185:342-9.

39. Gulliver A, Griffiths KM, Christensen $\mathrm{H}$ et al. Internet-based interventions to promote mental health help-seeking in elite athletes: an exploratory randomized controlled trial. J Med Internet Res. 2012;14:69.

40. Campbell-Sills L, Stein MB. Psychometric analysis and refinement of the connordavidson resilience scale (CD-RISC): Validation of a 10-item measure of resilience. $\mathrm{J}$ Trauma Stress. 2007;20:1019-28.

41. O'Connor M, Casey L. The Mental Health Literacy Scale (MHLS): a new scale-based measure of mental health literacy. Psychiatry Res. 2015;229:511-6.

42. Wilson CJ, Deane FP, Ciarrochi J, Rickwood D. Measuring help-seeking intentions: properties of the General Help-seeking Questionnaire. Canadian Journal of Counselling. 2005;39:15-28.

43. Yap MB, Mackinnon A, Reavley N, Jorm AF. The measurement properties of stigmatizing attitudes towards mental disorders: results from two community surveys. Int J Methods Psychiatr Res. 2014;23:49-61. 
44. Romero C, Master A, Paunesku D, Dweck CS, Gross JJ. Academic and emotional functioning in middle school: the role of implicit theories. Emotion. 2014;14(2):227-34.

45. Zimet GD, Powell SS, Farley GK, Werkman S, Berkoff KA. The multidimensional scale of perceived social support. J Pers Assess. 1998;52:30-41.

46. Mewton L, Kessler RC, Slade $\mathrm{T}$ et al. The psychometric properties of the Kessler Psychological Distress Scale (K6) in a general population sample of adolescents. Psychol Assess. 2016;28:1232-42.

47. Keyes CL. Brief description of the mental health continuum short form (MHC-SF) [Internet]. Atlanta, Georgia, USA. 2009 [cited 2020 Jun 06]. Available from: https://www.aacu.org/sites/default/files/MHC-SFEnglish.pdf

48. Australian Bureau of Statistics. Information paper. An introduction to Socio-Economic Indexes for Areas (SEIFA) 2006. Catalogue no. 2039.0. Canberra: Australian Bureau of Statistics. 2008.

49. Hurley DS, Swann C, Allen MS, Vella SA. A qualitative evaluation of a mental health literacy intervention for parents delivered through community sport clubs. Psychol Sport Exerc. 2020:47,101635.

50. Bapat S, Jorm A, Lawrence K. Evaluation of a mental health literacy training program for junior sporting clubs. Australas Psychiatry. 2009;17:475-9. 
51. Pierce D, Liaw S-T, Dobell J, Anderson R. Australian rural football club leaders as mental health advocates: an investigation of the impact of the Coach the Coach project. Int J Ment Health Sy. 2010;4:10.

52. Clarke AM, Kuosmanen T, Barry MM. A systematic review of online youth mental health promotion and prevention interventions. J Youth Adolesc. 2015;44:90-113.

53. Means B, Toyama Y, Murphy R, Baki M. The effectiveness of online and blended learning: a meta-analysis of the empirical literature. Teach Coll Rec. 2013;115(3):1-47.

54. Donaldson A, Leggett S, Finch CF. Sports policy development and implementation in context: researching and understanding the perceptions of community end-users. Int Rev Sociol Sport. 2011;47:743-60.

55. Klimes-Dougan B, Klingbeil DA, Meller SJ. The impact of universal suicide-prevention programs on the help-seeking attitudes and behaviors of youths. Crisis. 2013;34(2):82-97.

56. Maiuolo M, Deane FP, Ciarrochi J. Parental authoritativeness, social support and helpseeking for mental health problems in adolescents. J Youth Adolesc. 2019;48(6):1056-67.

57. Larzelere RE, Kuhn BR, Johnson B. The intervention selection bias: an underrecognized confound in intervention research. Psychol Bull. 2004;130:289-303.

58. Costigan CL, Cox MJ. Fathers' participation in family research: is there a self-selection bias? J Fam Psychol.15:706-20. 
59. Perry Y, Petrie K, Buckley H, Cavanagh L, Clarke D, Winslade M et al. Effects of a classroom-based educational resource on adolescent mental health literacy: a cluster randomised controlled trial. J Adolesc. 2014;37:1143-51. 
Table 1. Estimated marginal means, 95\% confidence intervals, and group x time interaction for all outcome measures in intention-totreat analyses.

\begin{tabular}{|c|c|c|c|c|c|}
\hline Variable & Time & $\begin{array}{c}\text { Intervention Group (95\% } \\
\text { Confidence Intervals) }\end{array}$ & $\begin{array}{l}\text { Control Group (95\% } \\
\text { Confidence Intervals) }\end{array}$ & $\begin{array}{c}\text { Adjusted Mean } \\
\text { Difference (95\% } \\
\text { Confidence Intervals) }\end{array}$ & $p$ \\
\hline \multirow[t]{2}{*}{ Depression Literacy } & Baseline & $6.70(6.24,7.16)$ & $5.92(5.61,6.23)$ & $-1.81(-2.39,-1.22)$ & $<.001$ \\
\hline & Follow up & $7.42(6.93,7.91)$ & $4.83(4.44,5.22)$ & & \\
\hline \multirow[t]{2}{*}{ Anxiety Literacy } & Baseline & $5.48(5.00,5.97)$ & $4.80(4.66,5.30)$ & $-2.04(-2.63,-1.45)$ & $<.001$ \\
\hline & Follow up & $6.52(6.01,7.04)$ & $3.98(3.58,4.37)$ & 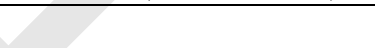 & \\
\hline \multirow[t]{2}{*}{ Social Distance } & Baseline & $3.47(3.33,3.60)$ & $3.49(3.40,3.58)$ & $-0.18(-0.34,-0.21)$ & .260 \\
\hline & Follow up & $3.62(3.48,3.76)$ & $3.47(3.35,3.58)$ & & \\
\hline \multirow[t]{2}{*}{ Helpseeking - Informal } & Baseline & $4.58(4.41,4.75)$ & $4.66(4.54,4.78)$ & $-0.11(-0.34,0.13)$ & .363 \\
\hline & Follow up & $4.65(4.47,4.84)$ & $4.62(4.47,4.78)$ & & \\
\hline \multirow[t]{2}{*}{ Helpseeking - Formal } & Baseline & $3.70(3.44,3.96)$ & $3.70(3.53,3.88)$ & $-0.40(-0.70,-0.11)$ & .008 \\
\hline & Follow up & $4.07(3.80,4.34)$ & $3.67(3.46,3.88)$ & & \\
\hline \multirow[t]{2}{*}{ Confidence to Seek Info } & Baseline & $4.07(3.91,4.23)$ & $3.87(3.77,3.98)$ & $-0.23(-0.44,-0.03)$ & .026 \\
\hline & Follow up & $4.32(4.15,4.49)$ & $3.89(3.76,4.02)$ & & \\
\hline \multirow[t]{2}{*}{ Resilience } & Baseline & $37.48(36.36,38.60)$ & $37.11(36.36,37.86)$ & $-2.09(-3.35,-0.84)$ & .001 \\
\hline & Follow up & $39.71(38.55,40.88)$ & $37.25(36.33,38.17)$ & & \\
\hline \multirow[t]{2}{*}{ Implicit Beliefs $^{\mathrm{a}}$} & Baseline & $10.30(9.82,10.77)$ & $10.29(9.97,10.61)$ & $0.65(-.00,1.31)$ & .051 \\
\hline & Follow up & $9.93(9.43,10.43)$ & $10.58(10.17,10.99)$ & & \\
\hline \multirow[t]{2}{*}{ Perceived Family Support } & Baseline & $5.98(5.79,6.18)$ & $5.95(5.82,6.08)$ & $-0.22(-0.45,0.01)$ & 0.60 \\
\hline & Follow up & $6.05(5.85,6.26)$ & $5.80(5.64,5.96)$ & & \\
\hline \multirow[t]{2}{*}{ Psychological Distress $^{\mathrm{a}}$} & Baseline & $5.12(4.50,5.73)$ & $4.92(4.52,5.33)$ & $0.36(-0.30,1.01)$ & .289 \\
\hline & Follow up & $4.60(3.96,5.24)$ & $4.76(4.28,5.24)$ & & \\
\hline \multirow[t]{2}{*}{ Wellbeing } & Baseline & $65.21(62.82,67.61)$ & $66.43(64.89,67.97)$ & $-3.71(-5.93,-1.48)$ & .001 \\
\hline & Follow up & $67.94(65.47,70.41)$ & $65.45(63.65,67.25)$ & & \\
\hline
\end{tabular}

Note. ${ }^{a}$ Decreases represent improvements over time. 
Table 2. Baseline means, standard deviations, and tests of baseline differences for all outcome measures between participants who completed the intervention per protocol and those who did not.

\begin{tabular}{|l|c|c|c|c|c|c|c|}
\hline \multirow{2}{*}{ Variable } & \multicolumn{2}{|c|}{$\begin{array}{c}\text { Completed Per Protocol } \\
(n=45)\end{array}$} & $\begin{array}{c}\text { Completed Less than Per } \\
\text { Protocol }(n=305)\end{array}$ & \multicolumn{3}{c|}{ Test of Baseline Differences } \\
\cline { 2 - 8 } & $M$ & $S D$ & $M$ & $S D$ & $t$ & $d f$ & $p$ \\
\hline Depression Literacy & 7.30 & 2.28 & 6.24 & 2.87 & -2.31 & 282 & .021 \\
\hline Anxiety Literacy & 5.95 & 2.16 & 5.12 & 2.62 & -2.00 & 283. & .046 \\
\hline Social Distance & 3.31 & 0.96 & 3.41 & 0.83 & 0.74 & 277 & .457 \\
\hline Helpseeking - Informal & 4.61 & 1.20 & 4.58 & 1.25 & -0.15 & 285 & .878 \\
\hline Helpseeking - Formal & 3.90 & 1.51 & 3.62 & 1.52 & -1.15 & 285 & .253 \\
\hline Confidence to Help & 4.20 & 0.89 & 4.01 & 0.85 & -1.37 & 275 & .171 \\
\hline Resilience & 39.64 & 5.19 & 37.45 & 6.56 & -2.09 & 279 & .038 \\
\hline Implicit Beliefs & 9.87 & 2.94 & 10.39 & 2.97 & 1.09 & 276 & .278 \\
\hline Perceived Family Support & 6.29 & 0.87 & 5.96 & 1.22 & $-2.17 *$ & 77 & .033 \\
\hline Psychological Distress & 4.39 & 2.83 & 4.93 & 3.26 & 1.04 & 285 & .297 \\
\hline Wellbeing & 70.74 & 9.18 & 65.37 & 11.69 & -2.82 & 267 & .005 \\
\hline
\end{tabular}

Note. *Equal variances not assumed. 
Table 3. Estimated marginal means, 95\% confidence intervals, and group x time interaction for all outcome measures in per-protocol analyses.

\begin{tabular}{|c|c|c|c|c|c|}
\hline Variable & Time & $\begin{array}{c}\text { Intervention Group: Per Protocol } \\
\text { (95\% Confidence Intervals) } \\
{[n=45]}\end{array}$ & $\begin{array}{c}\text { Control Group (95\% } \\
\text { Confidence Intervals) } \\
{[n=466]}\end{array}$ & $\begin{array}{l}\text { Adjusted Mean Difference } \\
\text { (95\% Confidence Intervals) }\end{array}$ & $p$ \\
\hline \multirow[t]{2}{*}{ Depression Literacy } & Baseline & $7.45(6.41,8.49)$ & $5.89(5.60,6.17)$ & $-1.77(-2.92,-0.63)$ & .003 \\
\hline & Follow up & $8.13(7.04,9.23)$ & $4.80(4.43,5.17)$ & 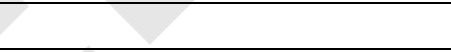 & \\
\hline \multirow[t]{2}{*}{ Anxiety Literacy } & Baseline & $6.00(4.88,7.11)$ & $4.96(4.65,5.28)$ & $-2.35(-3.54,-1.17)$ & $<.001$ \\
\hline & Follow up & $7.34(6.18,8.50)$ & $3.95(2.56,4.35)$ & 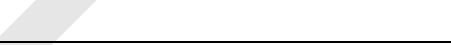 & \\
\hline \multirow[t]{2}{*}{ Social Distance } & Baseline & $3.47(3.17,3.76)$ & $3.48(3.39,3.56)$ & $-0.39(-0.70,-0.09)$ & .011 \\
\hline & Follow up & $3.84(3.52,4.15)$ & $3.45(3.35,3.56)$ & & \\
\hline \multirow[t]{2}{*}{ Helpseeking - Informal } & Baseline & $4.60(4.15,5.04)$ & $4.66(4.54,4.78)$ & $-0.13(-0.57,0.31)$ & .561 \\
\hline & Follow up & $4.69(4.22,5.15)$ & $4.62(4.46,4.77)$ & & \\
\hline \multirow[t]{2}{*}{ Helpseeking - Formal } & Baseline & $3.92(3.31,4.54)$ & $3.70(3.53,3.88)$ & $-0.37(-0.94,0.19)$ & .196 \\
\hline & Follow up & $4.26(3.63,4.90)$ & $3.67(3.45,3.88)$ & & \\
\hline \multirow[t]{2}{*}{ Confidence to Seek Info } & Baseline & $4.30(3.92,4.69)$ & $3.88(3.77,3.99)$ & $0.09(-0.32,0.50)$ & .673 \\
\hline & Follow up & $4.23(3.83,4.64)$ & $3.89(3.75,4.03)$ & & \\
\hline \multirow[t]{2}{*}{ Resilience } & Baseline & $39.36(36.97,41.75)$ & $37.18(36.51,37.85)$ & $-3.77(-6.24,-1.31)$ & .003 \\
\hline & Follow up & $43.33(40.81,45.84)$ & $37.37(36.51,38.23)$ & & \\
\hline \multirow{2}{*}{ Implicit Beliefs $^{\mathrm{a}}$} & Baseline & $9.74(8.70,10.77)$ & $10.33(10.04,10.63)$ & $2.48(1.16,3.79)$ & $<.001$ \\
\hline & Follow up & $7.54(6.43,8.64)$ & $10.60(10.22,10.99)$ & & \\
\hline \multirow[t]{2}{*}{ Perceived Family Support } & Baseline & $6.19(5.73,6.65)$ & $5.96(5.83,6.09)$ & $-0.18(-0.64,0.28)$ & .438 \\
\hline & Follow up & $6.22(5.74,6.70)$ & $5.81(5.65,5.97)$ & & \\
\hline \multirow[t]{2}{*}{ Psychological Distress $^{a}$} & Baseline & $4.35(3.00,5.69)$ & $4.94(4.56,5.32)$ & $1.29(-0.05,2.62)$ & .059 \\
\hline & Follow up & $2.88(1.48,4.28)$ & $4.76(4.30,5.22)$ & & \\
\hline \multirow[t]{2}{*}{ Wellbeing } & Baseline & $69.14(63.90,74.39)$ & $66.41(64.92,67.89)$ & $-6.21(-10.69,-1.74)$ & .007 \\
\hline & Follow up & $74.40(68.99,79.80)$ & $65.45(63.67,67.23)$ & & \\
\hline
\end{tabular}

Note. ${ }^{\mathrm{a}}$ Decreases represent improvements over time. 